\title{
A PROPOSED HYBRID AGILE FRAMEWORK MODEL FOR MOBILE APPLICATIONS DEVELOPMENT
}

\author{
Ammar Khader Almasri \\ ${ }^{1}$ Department of Management Information System, Albalqa Applied University, Amman, \\ Jordan
}

\begin{abstract}
With the increasing in mobile application systems and a high competition between companies, that led to increase in the number of mobile application projects.

Mobile software development is a group of process for creating software for mobile devices with limited resources like small screen, low-power. The development of mobile applications is a big challenging because of rapidly changing business requirements and technical constraints for mobile systems. So, developers faced the challenge of a dynamic environment and the Changing of mobile application requirements. Moreover, Mobile applications should adapt appropriate software development methods that act in response efficiently to these challenges.
\end{abstract}

However, at the moment, there is limited knowledge about the suitability of different software practices for the development of mobile applications. According to many researchers, Agile methodologies was found to be most suitable for mobile development projects as they are short time, require flexibility, reduces waste and time to market.

Finally, in this research we are looking for a suitable process model that conforms to the requirement of mobile application, we are going to investigate agile development methods to find a way, making the development of mobile application easy and compatible with mobile device features.

\section{KEYWORDS}

Mobile Application Development process, Agile Methodologies, CMMI.

\section{INTRODUCTION}

\subsection{Traditional \& Classical Methodologies}

Software methodologies like Waterfall, Rapid Application Prototype, Spiral, Rational Unified Process, and V-shaped are called traditional software development methodologies and these are called the heavyweight methodologies [1]. These methodologies are based on a sequential series of steps. 


\subsubsection{Waterfall Process Model}

The Classical Life Cycle or the Waterfall Process Model [2] was proposed by Winston W. Royce in 1970. The first process model to present sequential steps, in this model the work is done in linear fashion.

\subsubsection{Rapid Application Development (RAD) Model}

This model was developed by IBM in 1980. In this model a rapid prototype is built up and given to user for evaluation \& obtaining feedback. Then on the basis of user feedback Prototype is refined.

\subsubsection{Spiral Model}

Spiral model was developed by Barry Boehm in 1986. In this model the work is done like Waterfall Process Model through a sequence of activities with some backtracking between activities[3].this model mixes characteristics of both prototype model and waterfall process model.

\subsubsection{Rational Unified Process (RUP) model}

Rational Unified Process is one of the most popular of object oriented software development processes. RUP is an iterative approach which is Maintained and developed by Rational Software Corporation. The software lifecycle is broken into cycles the system will grow incrementally over time, cycle by cycle.

\subsubsection{Shaped Model}

The V-model just like the waterfall model, the V-Shaped life cycle is a sequential path of execution of processes. Each phase must be completed before the next phase begins. The Vmodel establishes the relationships between each phase of the development life cycle and its associated phase of testing. V- Model is simple and easy to use.

\subsubsection{Agile Methodology}

Agile development methods referred to lightweight approaches [4]. Agile development is used the incremental and iterative work strategy, the development life cycle is divided into smaller parts as well as revisited over and over again. It iteratively improves software based on customer feedback [5]. According to [6], Agile models are used to build a project if the project has the following aspects: (1) project's requirement changes frequently (2) project is small (3) needs to deliver product in short period time. 
There are a number of software development methodologies that have been categorized under the Manifesto umbrella. XP, SCRUM, DSDM, FDD, and Crystal Methods are among the most popular examples of these agile methods. This research will view the following five models:

1. Extreme Programming (XP)

2. Scrum

3. Crystal

4. Dynamic Systems Development Method (DSDM)

5. Feature-Driven Development (FDD)

\subsubsection{Extreme Programming (XP)}

Extreme Programming (XP) was launched in 1999 by Kent Beck, Ward Cunningham and Ron Jeffries for small to medium teams that develop software products with changing requirements[7]; XP is one of a lightweight and iterative informal software development methodology[8].

- It is suited for projects with dynamic requirements[9]

- It is suitable for small to medium teams that develop software products with changing requirements as well as collaboration within the team[10].

- It has weekly cycle and continuous integration[7]

- It has designed for development teams of between 3 and 10[11] .

\subsubsection{Scrum}

The method was proposed in details by Schwaber and Beedle [12]; is an iterative and incremental method whose purpose is to help development teams to concentrate on established goals and minimize the work done on less important tasks.

- Keep the simplicity in a complicated business environment and if the requirements are unclear \& ambiguous[13].

- a development team should interact to create a flexible, adaptive and productive system in a constantly changing environment.

- feed-back loops are the main element [12]

\subsubsection{Crystal Methodologies}

Crystal clear is one of agile software development a methodology was developed by Alistair Cockburn. This method has many methodologies based on complexity like size and type of the team (Clear, Yellow, Orange, Red, Maroon, Blue, and Violet). The most of these agile methods are Crystal Clear that concentrate on communication between small teams that develop noncritical software. 
- It can be applied to teams having up to 6 developers[11].

- It can be applied to small teams and small projects that are not life critical[11].

\subsubsection{Dynamic Software Development Method (DSDM)}

DSDM is a public domain Rapid Application Development method which has been developed over many years by the members of a nonprofit organization called the DSDM Consortium based on their experiences, which aimed to deliver software quickly through prototyping and iterative design and testing.

- Active user involvement is necessary[14, 15].

- The main concern is on frequent delivery of products[14, 15].

- Suitability for business purpose is the essential criteria for acceptance of deliverable[14, 15].

\subsubsection{Feature-Driven Development}

Feature Driven Development (FDD) was founded by Jeff De Luca and Peter Coad. Combines model driven development with agile development, emphasizing the initial object model, work division into features and iterative design of each feature. Claims to be best suited for critical system development. An iteration of a feature has two stages: design and development [16].

- This method primarily focuses on design and implementation of high quality deliverables.

\section{MOBILE SOFTWARE DEVELOPMENT}

With the increasing in mobile application systems and a high competition between companies, that led to increase in the number of mobile application projects. Mobile software development is a group of process for creating software for mobile devices with limited resources like small screen, low-power. The development of mobile applications is a big challenging because of rapidly changing business requirements and technical constraints for mobile systems. So, developers faced the challenge of a dynamic environment and the Changing of mobile application requirements. Moreover, Mobile applications should adapt appropriate software development methods [17].

Many studies have been suggested a solution to the above challenges, it has been suggested that Agile is a fit model for the development of mobile applications [18].Moreover, There are a set of characteristics were developed by [19] regarding using agile methodology for mobile application development as follow :(1) High environment volatility(2)Small development teams (3) Identifiable customer (3) Object oriented development (4) Non-safety critical Software (5)Application level Software(6) Small systems (7) and Short development cycles. 
However, the quality, quantity, and limited understanding about the appropriateness of different software practices for mobile applications development open new directions for researchers in computer science and industry to find a model to be fit for mobile applications development; Agile software development practice have given attention of software developers and researchers during the last decade but scientific research and published outcomes still remains quite limited[17].

\section{Problem STATEMENT}

Software is integrated in a vast amount of products like cars, entertainment, and mobile phones and is a major success factor determining whether a product succeeds[20].

A Successful for any software is related to delivery that software with high quality software. The key success factor to deliver any software products with high quality outcome is picking up appropriate and well-working software development process[21]. Moreover, mobile applications like other desktop applications need appropriate and well-working software development process.

Nevertheless, mobile applications have different features which limit their performance and efficiency like application size, mobile hardware features (memory size, CPU clock rate, platform type, data representation, and battery life).

Developers for mobile application software face the following challenges:

(1) a dynamic environment with frequent modifications in customer needs and expectations[17].

(2) technical constraints associated with mobile systems [17].

(3) different technological platforms, including different Operating Systems (OS) and `software development platforms [22].

(4) There is limited research to find a suitable model for the development of mobile applications[17].

Traditional software development process approaches that used in the development of desktop application may not to be directly applicable to mobile application[23] .therefore, it is still critical to adopt appropriate methodology for the development of mobile application[24]. So, mobile applications development needs to have customized development processes [18, 25] to suite the special mobile application requirements with limited resources of mobile devices as well as to meet customer needs and to deliver fast to the market.

However, at the moment, there is limited knowledge about the suitability of different software practices for the development of mobile applications[17]. Finally, in this research we are looking for a suitable process model that conforms to the requirement of mobile application, we are going to investigate agile development methods to find a way, making the development of mobile 
application easy and compatible with mobile device features. Agile methodologies was found to be most suitable for mobile development projects as they are short time, require flexibility, reduces waste and time to market[17].

\section{RELATED RESEARCH}

Many studies have been conducted d on mobile applications development by using Agile methodologies to propose as an appropriate framework.

In 2008 a study was investigated by Rahimian and Ramsin [26] to propose as an appropriate framework for the development of mobile systems based on of Agile and risk-based methodologies which called Hybrid Method Engineering (HME). It is based on a combination of agile methodologies, ASD and New Product Development (NPD). The outcome of this research paper is the Hybrid Methodology Design Process. The research doesn't include a case study or experimental setup that tests this methodology.

A study was conducted by Scharff and Verma [27], to prove the efficiency of Scrum for the development of mobile applications. The authors propose to use Scrum as a model for mobile software development. This study was based on 1 case study not related to mobile software.

Scrum Lean Six Sigma (SLeSS) is a new approach was developed by da Cunha, et al. [28] for the development of embedded software for mobile. This model is integration between Scrum and Lean Six Sigma. Authors propose to plan the implementation of Scrum alone, following this, it is necessary to apply organizational adjustments to implement LSS. This study implemented in only one mobile software company that is not a report of its utilization. This proposed model enables the reaching of performance and a high quality, improving the development process and the project's outcome.

This study was investigated by Abrahamsson [19] , to map agile home ground themes with mobile software development the result indicted that agile innovations present a solution for mobile application developers with high quality development processes.

A broad online survey was conducted by Flora, et al. [17] in 2014 to find out which agile approaches is suitable for mobile applications. The questionnaire for this study was assessed and validated by several experts in Agile models; around 130 participants (development team members, Agile experts, researchers, and other stakeholders) were responded. The results indicate that Agile methodologies of course fits and suitable for mobile development projects based on the following factors short time, require flexibility, and reduces time to market.

According to prior studies were developed to find appropriate software development model for mobile applications development the researchers are agreed with that (1) agile methodologies is one of the most suitable model for mobile development (2) these Agile methods are highly helpful tools to deliver enhanced speed and high quality for mobile applications development. 


\section{RESEARCH METHODOLOGY:}

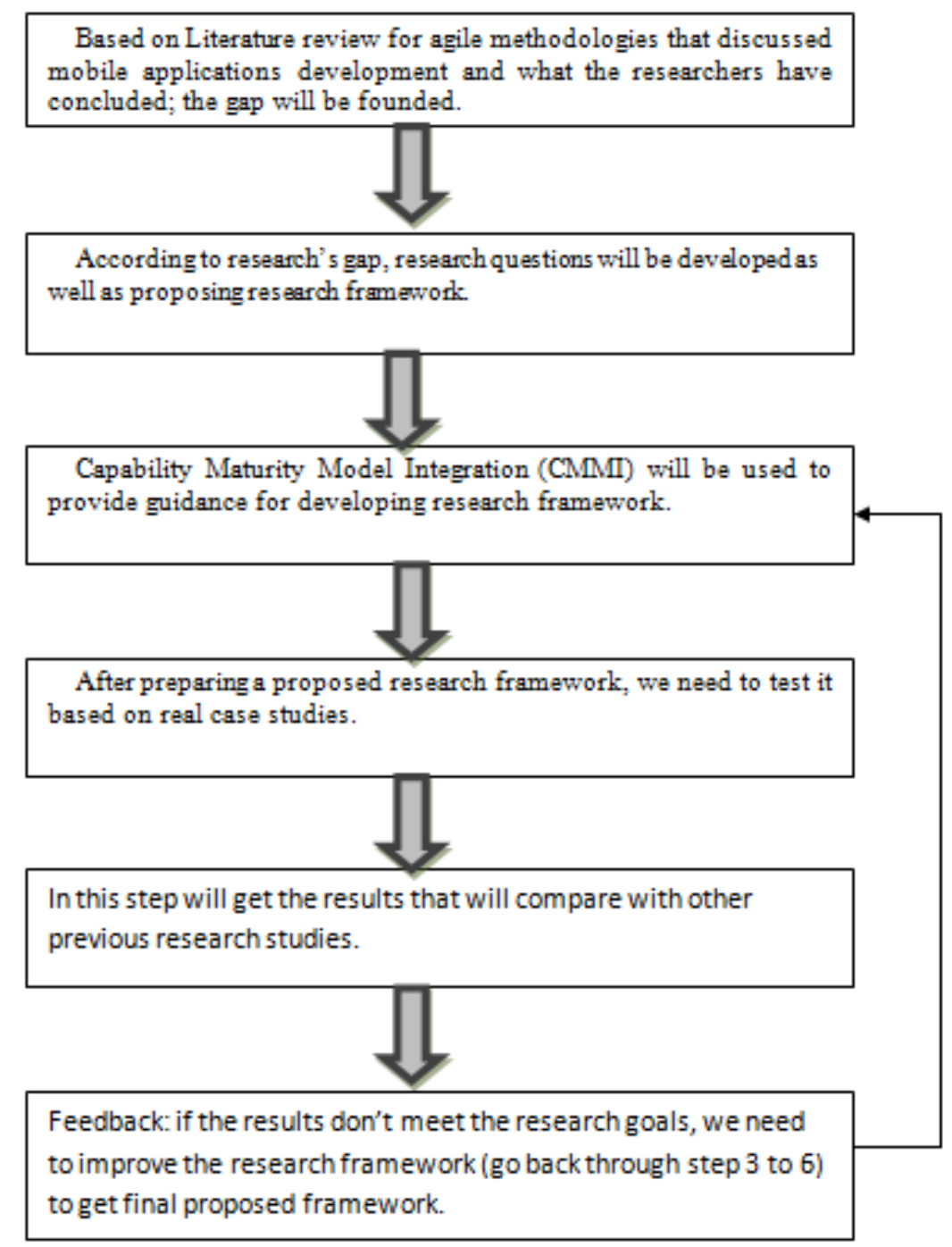

\subsection{RESEARCH QUESTIONS}

1- Are agile methodologies effects positively on mobile application development?

2- Are agile methodologies with CMMI effects positively on mobile application development?

3- Does proposed model affect positively on mobile application development? 
International Journal of Software Engineering \& Applications (IJSEA), Vol.7, No.2, March 2016

4- Does the expected outcome have a significant effect on mobile application development?

\section{CONCLUSION}

According to literature review, there is no research study that provides detail reasons to use Agile as essential model for mobile application development for that reason, this research aims to encourage and contribute to prove that as well as helping developers in using a standardized model for developing mobile applications based on agile methodologies.

\section{REFERENCES}

[1] O. Nikiforova, V. Nikulsins, and U. Sukovskis, "Integration of MDA Framework into the Model of Traditional Software Development," in DB\&IS, 2008, pp. 229-239.

[2] W. W. Royce, "Managing the development of large software systems," in proceedings of IEEE WESCON, 1970.

[3] R. J. Madachy, Software process dynamics: John Wiley \& Sons, 2007.

[4] G. Milanov and A. NJEGUŠ, "Analysis of Return on Investment in Different Types of Agile Software Development Project Teams," Informatica Economica, vol. 16, pp. 7-18, 2012.

[5] Y. B. Leau, W. K. Loo, W. Y. Tham, and S. F. Tan, "Software Development Life Cycle AGILE vs Traditional Approaches," in International Conference on Information and Network Technology, 2012, pp. 162-167.

[6] S. Balaji and M. Murugaiyan, "Waterfall vs. V-Model vs. Agile: A comparative study on SDLC," International Journal of Information Technology and Business Management, vol. 2, pp. 26-30, 2012.

[7] C. Andres and K. Beck, "Extreme Programming Explained: Embrace Change," Reading: AddisonWesley Professional, 2004.

[8] M. Abouelela and L. Benedicenti, "Bayesian Network Based XP Process Modelling," arXiv preprint arXiv:1007.5115, 2010.

[9] M. Stoica, M. Mircea, and B. Ghilic-Micu, "Software Development: Agile vs. Traditional," Informatica Economica, vol. 17, pp. 64-76, 2013.

[10] H. Svensson and M. Host, "Introducing an agile process in a software maintenance and evolution organization," in Software Maintenance and Reengineering, 2005. CSMR 2005. Ninth European Conference on, 2005, pp. 256-264.

[11] G. Kumar and P. K. Bhatia, "Comparative Analysis of Software Engineering Models from Traditional to Modern Methodologies," in Advanced Computing \& Communication Technologies (ACCT), 2014 Fourth International Conference on, 2014, pp. 189-196.

[12] K. Schwaber and M. Beedle, "gilè Software Development with Scrum," 2002.

[13] G. Ahmad, T. R. Soomro, and M. N. Brohi, "XSR: Novel Hybrid Software Development Model (Integrating XP, Scrum \& RUP)."

[14] A. Sani, A. Firdaus, S. R. Jeong, and I. Ghani, "A review on software development security engineering using dynamic system method (DSDM)," International Journal of Computer Applications, vol. 69, pp. 33-44, 2013.

[15] J. Stapleton, DSDM: Business focused development: Pearson Education, 2003.

[16] S. R. Palmer and M. Felsing, A practical guide to feature-driven development: Pearson Education, 2001.

[17] H. K. Flora, X. Wang, and S. Chande, "Adopting an Agile Approach for the Development of Mobile Applications," International Journal of Computer Applications, vol. 94, pp. 43-50, 2014. 
International Journal of Software Engineering \& Applications (IJSEA), Vol.7, No.2, March 2016

[18] R. Holler, "Mobile application development: a natural fit with agile methodologies," VerisonOne LLC, Alpharetta, 2006.

[19] P. Abrahamsson, "Keynote: Mobile software development-the business opportunity of today," in Proceedings of the International Conference on Software Development, 2005, pp. 20-23.

[20] K. Petersen and C. Wohlin, "Issues and advantages of using agile and incremental practices," Software Engineering Research and Practice, 2008.

[21] S. a. J. Khalaf and M. N. Al-Jedaiah, "Software quality and assurance in waterfall model and XP: a comparative study," WSEAS Transactions on Computers, vol. 7, pp. 1968-1976, 2008.

[22] A. Hammershoj, A. Sapuppo, and R. Tadayoni, "Challenges for mobile application development," in Intelligence in Next Generation Networks (ICIN), 2010 14th International Conference on, 2010, pp. 1-8.

[23] A. I. Wasserman, "Software engineering issues for mobile application development," in Proceedings of the FSE/SDP workshop on Future of software engineering research, 2010, pp. 397-400.

[24] H. K. Flora, X. Wang, and S. V. Chande, "An Investigation into Mobile Application Development Processes: Challenges and Best Practices," International Journal of Modern Education and Computer Science (IJMECS), vol. 6, p. 1, 2014.

[25] H. S. Alamri and B. A. Mustafa, "Software Engineering Challenges in Multi Platform Mobile Application Development," Advanced Science Letters, vol. 20, pp. 2115-2118, 2014.

[26] V. Rahimian and R. Ramsin, "Designing an agile methodology for mobile software development: a hybrid method engineering approach," in Research Challenges in Information Science, 2008. RCIS 2008. Second International Conference on, 2008, pp. 337-342.

[27] C. Scharff and R. Verma, "Scrum to support mobile application development projects in a just-in-time learning context," in Proceedings of the 2010 ICSE Workshop on Cooperative and Human Aspects of Software Engineering, 2010, pp. 25-31.

[28] T. F. V. da Cunha, V. L. L. Dantas, and R. M. Andrade, "SLeSS: A Scrum and Lean Six Sigma integration approach for the development of sofware customization for mobile phones," in Software Engineering (SBES), 2011 25th Brazilian Symposium on, 2011, pp. 283-292. 